\title{
The Effect of Parathyroid Hormone on Osseointegration in Insulin-Treated Diabetic Rats
}

\author{
Tina Rybaczek, DDS, * Stefan Tangl, MSc,† Toni Dobsak, BSc, † Reinhard Gruber, PhD,§ and \\ Ulrike Kuchler, MD, DMDף
}

$\mathrm{D}$ iabetes mellitus is the most common endocrine disorder, and the World Health Organization has estimated the number of diabetic patients rising up to 366 million in the year 2030., ${ }^{1,2}$ Elevated blood glucose levels are a hallmark of diabetes mellitus. Diabetic patients have a high risk of developing susceptibility to infections and delayed healing, atherosclerosis associated with myocardial infarction and coronary artery disease, or neuropathy strokes. ${ }^{3,4}$ Beside these life-threatening complications, diabetes affects bone metabolism that leads to changes in bone structure, mineral density, and the extent of their contribution to the increase of fracture risk. ${ }^{5-7}$

Uncontrolled diabetes is also a risk factor in implant dentistry. ${ }^{7-9}$ Implant loss in patients with uncontrolled diabetes mellitus is significant, especially within the early phase of osseointegration. ${ }^{9,10}$

*Assistant Doctor, Department of Oral Surgery, Medical

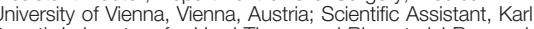
Donath Laboratory for Hard Aissue and Blomaterial Research, Vienna, Austria; Austrian Cluster for Tis

tHead, Karl Donath Laboratory for Hard Tissue and Biomaterial

奋searchtific Assistant, Karl Donath Laboratory for Hard Tissue and Biomaterial Research, Vienna, Austria.

and Blomaterial Research, Vienna, Austria. 1 Hniversity of \$Head, Department of Oral Biology, Medical University of Preventive Restorative, and Pediatric Dentistry, University of Bern, Bern, Switzerland.

IAssistant Professor, Department of Oral Surgery, Medica Department of Oral Surgery and Stomatology, University of Bern, Bern, Switzerland.

Reprint requests and correspondence to: Reinhard Gruber, PhD, Department of Oral Biology, Medical University of Vienna, Vienna, Austria, E-mail: reinhard. gruber@meduniwien.ac.at

ISSN 1056-6163/15/02404-392

Implant Dentistry

Volume 24 - Number 4

Copyright $(2015$ Wolters Kluwer Health, Inc. All rights

reserved.

DOI: 10.1097/ID.0000000000000288
Objectives: Uncontrolled diabetes mellitus is associated with impaired osseointegration. Diabetic individuals might benefit from bone anabolic therapies. Intermittent administration of 1-34 parathyroid hormone (PTH) stimulates bone formation in rodent models. However, this anabolic effect fails in diabetic rats. Whether the anabolic effect of PTH can be achieved in insulincontrolled diabetic rats has not been investigated yet.

Materials and Methods: After diabetes induction with streptozotocin in 40 female Wistar rats, the animals were randomly divided into 4 groups: diabetes, diabetes plus PTH, insulin-treated diabetes, and insulin-treated diabetes plus PTH. After 1 week, miniscrews were inserted in the tibiae. Osmotic pumps with insulin or saline solution were

Blood glucose levels seemingly affect the early implant stability, which is a surrogate parameter of osseointegration. ${ }^{11}$ One strategy is strict glycemic controls, but many patients exceed the therapeutic range. ${ }^{12}$ Consequently, therapies to improve the early stages of osseointegration in diabetic patients are demanding.

Parathyroid hormone (PTH) has attracted interest as a bone anabolic agent and is approved for the therapy of osteoporosis. ${ }^{13}$ In addition to the beneficial effect of PTH on bone implanted. Animals received 60 $\mathrm{mg} / \mathrm{kg}$ PTH or saline solution. Histomorphometric analysis was performed.

Results: In diabetic rats, no changes of medullary periimplant bone area or bone-to-implant contacts (BICs) were achieved with or without treatment with PTH. However, also animals treated with insulin failed to response significantly to PTH regarding bone area (7.4 \pm $4.1 \%$ and $8.1 \pm 4.1 \%)$ and BICs $(33.7 \pm 16.9 \%$ and $49.9 \pm 11.9 \%)$.

Conclusion: These results demonstrate that the metabolic characteristics of the diabetic rats produced a condition unable to respond to PTH treatment, even when hyperglycemia was controlled with insulin. (Implant Dent 2015;24:392-396)

Key Words: bone, teriparatide, rodent model, hyperglycemia

remodeling, preclinical studies have demonstrated the positive effect of PTH on fracture healing. ${ }^{14-20}$ Recently, also clinical evidence that PTH supports fracture healing became available. ${ }^{21,22} \mathrm{~A}$ recent clinical study showed the positive effect of PTH on bone regeneration after periodontal surgery. ${ }^{23}$ Moreover, PTH has been shown to stimulate the process of osseointegration in preclinical models, ${ }^{20,24}$ and clinical pilot studies were also performed. ${ }^{25}$ The spectrum of indication for the administration of PTH 


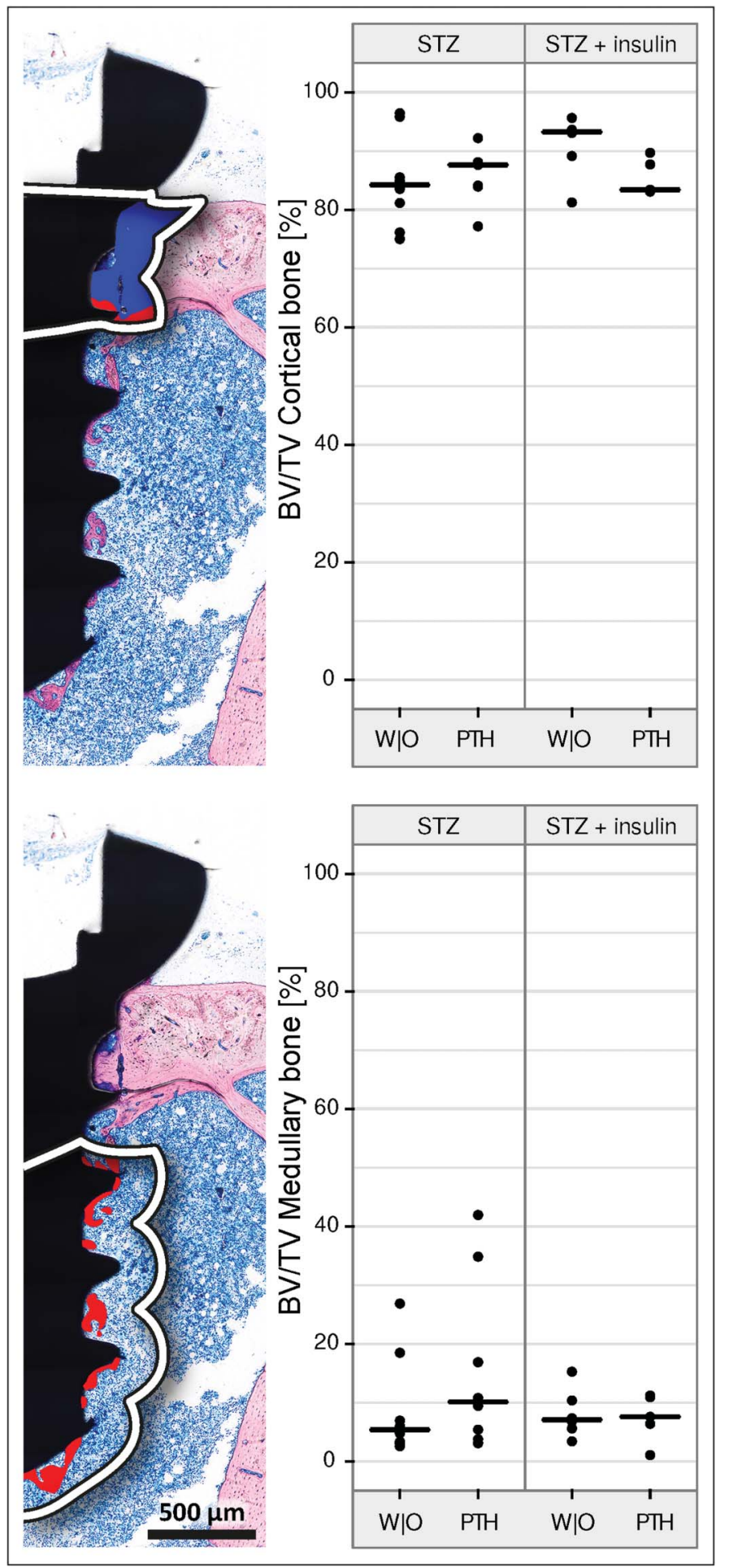

Fig. 1. Periimplant bone area (BV/TV): Medullary and cortical compartments. Type 1 diabetes was induced with intraperitoneal streptozotocin (STZ). Insulin was applied through osmotic pumps. The pump was filled with insulin (Humulin R [100 IU/mL] providing approximately 2 units per day; Eli Lilly and Company). Rats received $60 \mathrm{mg}$ PTH per kilogram body weight 3 times per week for 4 weeks.

is rising, but it remains unknown how metabolic changes influence the effect of PTH.
A recent study has shown that PTH fails to enhance osseointegration in an experimental type 1 diabetes model. ${ }^{26}$
Thus, the general held view that intermittent administration of PTH improves the process of bone regeneration not necessarily accounts true for compromised situations in diabetic patients. This has prompted us to suggest that PTH can exert its anabolic function when type 1 diabetes is controlled by insulin. We therefore tested the effect of PTH osseointegration in type 1 diabetic rats treated with insulin.

\section{Materials ANd Methods}

\section{Study Design, Ethics, and Induction of Diabetes}

Forty 6-month-old female Wistar rats from the Division for Biomedical Research (Himberg, Austria) were used for this study. The animals were treated according to the guidelines for animal care with free access to water and a standard diet. The rats were divided into 4 groups: diabetes, diabetes and PTH, insulin-treated diabetes, and insulin-treated diabetes with PTH. The type 1 diabetes was induced with a single intraperitoneal dose of $45 \mathrm{mg} / \mathrm{kg}$ streptozotocin (STZ; Sigma, St. Louis, MO) in citrate buffer. Weights and blood glucose levels were monitored at the day of implantation and at the day of being killed. Rats were considered as diabetic, if blood glucose levels exceeded $200 \mathrm{mg} / \mathrm{dL}$. The Medical University of Vienna Ethical Review Board for Animal Research approved the study protocol (GZ 66.009/0275-II/ 10b/2008).

\section{Surgical Procedures and \\ Postoperative Treatment}

One week after diabetes induction, the implant surgery was performed. Before the surgery, all animals received ketamine $(100 \mathrm{mg} / \mathrm{kg}$; Pharmacia \& Upjohn, Erlangen, Germany) and xylazine hydrochloride $(5 \mathrm{mg} / \mathrm{kg}$; Bayer, Leverkusen, Germany) by intramuscular injection. An incision was made over the proximal metaphysis of the left tibia, and muscles and periosteum were displaced. Ten millimeters below the knee joint, a hole was drilled and a titanium miniscrew (diameter $1 \mathrm{~mm}$, length $3 \mathrm{~mm}$; Medos Medizintechnik, Vienna, Austria) was inserted. To install the osmotic pumps (Osmotic pump Model 2ML4; Alzet Technical 


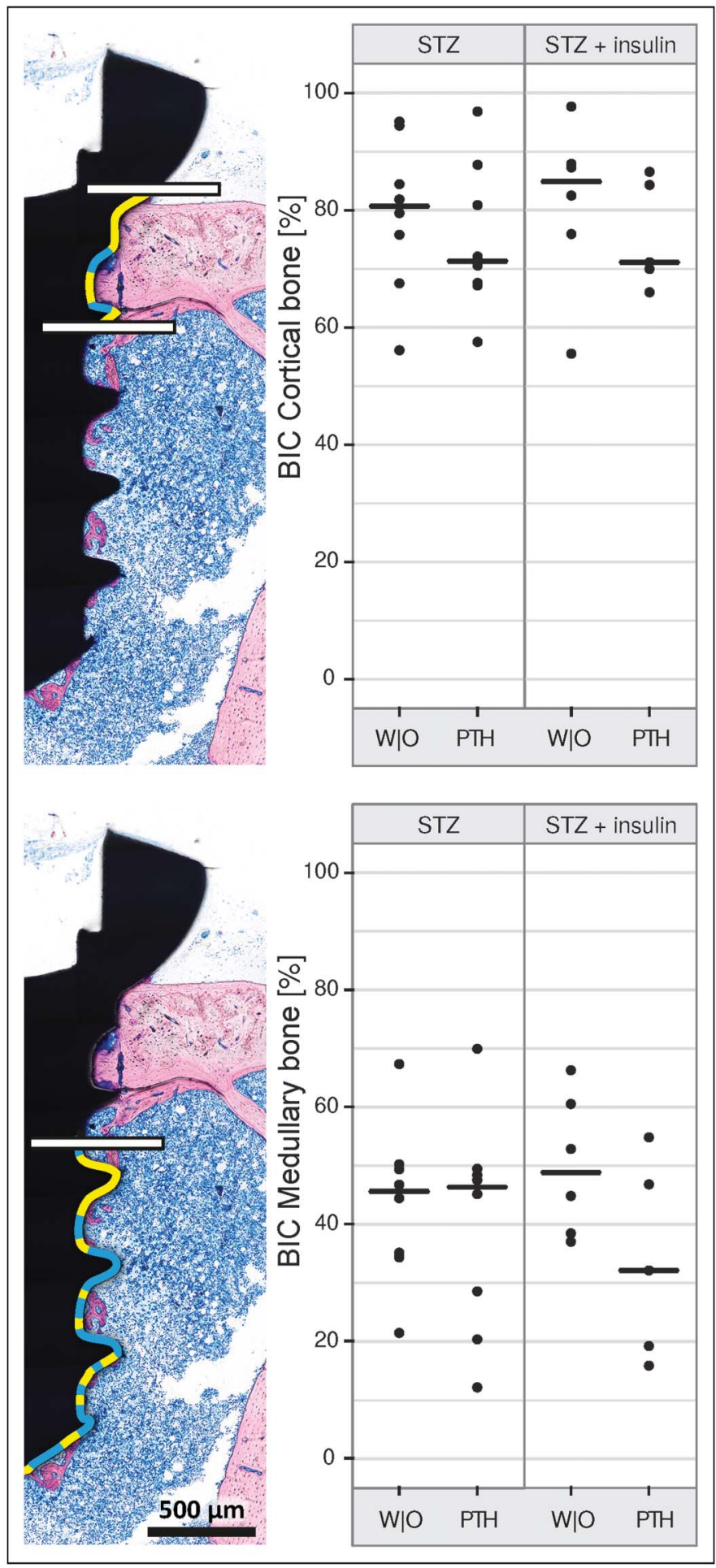

Fig. 2. Bone-to-implant contact (BIC): Medullary and cortical compartments. Type 1 diabetes was induced with intraperitoneal streptozotocin (STZ). Insulin was applied through osmotic pumps. The pump was filled with insulin (Humulin R $[100 \mathrm{IU} / \mathrm{mL}]$ providing approximately 2 units per day; Eli Lilly and Company). Rats received $60 \mathrm{mg}$ PTH per kilogram body weight 3 times per week for 4 weeks.

Support, DURECT Corporation, Cupertino, CA), a horizontal incision was made at the dorsal neck, and by blunt dissection, the subcutaneous tissues between the shoulder blades were expanded to create space for the osmotic pump. The pumps were filled with insulin (Humulin R [100 IU/mL] providing approximately 2 units per day; Eli Lilly and Company USA, LLC, Indianapolis, IN) or physiologic saline solution. Wounds were closed with resorbable sutures (Vicryl5-0; Ethicon $\mathrm{GmbH}$, Norderstedt, Germany). Butorphanol $(0.05 \mathrm{mg} / \mathrm{kg}$; Fort Dodge Laboratories, Girona, Spain) was administered for pain relief. A sterile solution of $60 \mathrm{mg}$ recombinant rat 1-34 $\mathrm{PTH}$ (\#H5460; Bachem, Bubendorf, Switzerland) per $1 \mathrm{~mL}$ vehicle $(10 \mathrm{mM}$ citric acid and $2 \%$ heat inactivated rat serum) was prepared weekly and stored at $4{ }^{\circ} \mathrm{C}$ until use. Three times per week, rats received 60 mg PTH per kilogram body weight or a vehicle by subcutaneous injection, starting at the day of implant placement. After 4 weeks, the animals were killed by intraperitoneal injection of thiopental (150 mg/kg; Nycomed, Konstanz, Germany).

\section{Sample Processing}

The tibiae were removed and fixed in phosphate-buffered formalin (RotiHistofix 4\%; Carl Roth, Karlsruhe, Germany). The samples were dehydrated with ascending alcohol grades and embedded in light-curing resin (Technovit 7200 VLC + BPO; Kulzer \& Co., Wehrheim, Germany). Blocks were further processed by using EXAKT cutting and grinding equipment (Exakt Apparatebau, Norderstedt, Germany) to thin ground sections along the implant axis. The sections were stained according to the method of Levai-Laczko. Sections were scanned with Olympus BX61VS microscope (Olympus Deutschland, Hamburg, Germany). Medullary and cortical compartments in an area of $200 \mu \mathrm{m}$ around the screw were defined as regions of interest (ROI). The images were analyzed based on rule sets considering the color and shape of the structures in the ROI with Definiens Developer XD2.0 (Definiens, Munich, Germany) followed by manual control using Photoshop (Adobe, San Jose, CA).

\section{Histomorphometric Analysis}

Within the $200 \mu \mathrm{m}$ of the ROI, 2 parameters were recorded: The percentage of present bone in the ROI was 
determined as "bone volume per tissue volume" (BV/TV), and screw surface in contact with bone was called "bone-toimplant contact" (BIC) and calculated as a percentage. Although this bone area is a 2-dimensional measurement, it is proportional to the 3-dimensional volume of bone. The medullary and cortical compartments were analyzed separately according to $\mathrm{BV} / \mathrm{TV}$ and BIC (Figs. 1 and 2). The proximal 400 $\mu \mathrm{m}$ of the medullary compartment was excluded of the analysis because of possible drilling dust. ${ }^{26}$ Screws with close proximity to the cortical bone were also excluded of the study because these situations can act as an osteoconductive bone graft on the local bone, irrespective of the treatment.

\section{Statistics}

Mean values of $\mathrm{BV} / \mathrm{TV}$ and $\mathrm{BIC}$ were compared in the medullary and cortical compartments of diabetic rats or animals treated with PTH by twoway analysis of variance on the data. In case of a significance, post hoc multiple $t$-tests were conducted to test the PTH effect, both in the control and the diabetes group. The Benjamini-Hochberg procedure was applied to correct for multiple testing, and significance was assigned at the $5 \%$ level.

\section{RESUlts}

\section{Effects of STZ and Insulin Treatment}

Blood glucose levels beyond 200 $\mathrm{mg} / \mathrm{dL}$ are a determinant of diabetes. The blood glucose levels of the animals rose after STZ administration up to an average value of $431 \mathrm{mg} / \mathrm{dL}$ within a week and up to $579 \mathrm{mg} / \mathrm{dL}$ throughout the experiment. Due to the insulin treatment, the blood glucose levels in the medicated rats fell again to normal values (mean: $175 \mathrm{mg} / \mathrm{dL}$ ).

\section{Evaluation of PTH Effect on \\ Osseointegration in the \\ Medullary Compartment}

As previously reported, PTH increases the BV/TV in a normoglycemic group but not in type 1 diabetic rats. ${ }^{26}$ Also in this study, no increase of medullary BV/TV was achieved in diabetic rats treated with PTH $(15.8 \pm 14.8 \%$ and 9.0 $\pm 8.6 \%)$. The same applies true for BIC
$(43.6 \pm 13.6 \%$ and $40.2 \pm 18.7 \%)$. New, however, is that type 1 diabetic rats treated with insulin showed no increase in BV/TV or BIC under PTH treatment $(8.1 \pm 4.1 \%$ and $7.4 \pm 4.1 \% ; 49.9 \pm$ $11.9 \%$ and $33.7 \pm 16.9 \%$, respectively). Thus, insulin alone cannot reverse susceptibility of type 1 diabetic rats to respond to PTH in a tibia implantation model.

\section{Evaluation of PTH Effect on \\ Osseointegration in the \\ Cortical Compartment}

In support of previous observation in normoglycemic and type 1 diabetic rats, ${ }^{24,26,27}$ the cortical BC/TV was similar in all treatment groups: diabetes, diabetes plus PTH, insulin-treated diabetes, and insulin-treated diabetes plus PTH $(84.8 \pm 7.9 \%, 86.1 \pm 4.4 \%, 91.0 \pm$ $5.2 \%$, and $85.4 \pm 3.1 \%$, respectively). The same accounts true for BICs where PTH had no considerable impact on osseointegration in diabetic rats that remain untreated or when glucose levels were controlled with insulin (79.4 \pm $13.0 \%, 75.1 \pm 12.7 \%, 81.2 \pm 14.4 \%$, and $75.6 \pm 9.2 \%$, respectively).

\section{DisCUSSION}

Based on our previous observations that type 1 diabetes lowers the impact of PTH on medullary osseointegration, ${ }^{26}$ we hypothesized that correcting blood glucose make animals susceptible for the anabolic effects of PTH. The main finding of this study was, however, that PTH failed to improve osseointegration in diabetic rats treated with insulin. These observations are important as they suggest that correcting blood glucose alone is not enough make the animals susceptible for PTH in the present model.

If we relate our findings to those of others, our data are in line with previous data showing around 15\% medullary $\mathrm{BV} / \mathrm{TV}$ in control and STZ-treated rats. Surprisingly, however, previous data showed approximately $15 \%$ medullary BIC, whereas here we have measured around $40 \% \mathrm{BIC}$, indicating that the models cannot directly be compared. The underlying reasons remain unknown. This study can, however, confirm previous observations that
PTH fails to support osseointegration in type 1 diabetic rats, ${ }^{26}$ possibly involving deterioration of the insulinlike growth factor 1 (IGF-1) levels. ${ }^{28-30}$ In this study, insulin pumps lowered the blood glucose to almost normoglycemic conditions. However, since we have not measured the impact of insulin on the IGF-1 serum levels, we have to consider this as a study limitation. It is thus possible that IGF-1 levels are still below the threshold required to exert the anabolic effects of PTH in vivo.

The clinical relevance of the present finding has to be interpreted with care as the model only partially reflects the situating in patients. Obviously, rodent models heal faster than humans, the tibia is not the alveolar bone, and the uncontrolled type 1 diabetes with the massive hyperglycemia is a rare situation. Clearly, this is a proof of principles study with one observation period. Thus, it cannot be ruled out that at other time points, PTH might have supported osseointegration in the insulin-treated rat models, for example, when considered that after 4 weeks, almost half of the implant surface is covered by bone. Overall, the data provide further support for the idea that severe diabetes, even when partially controlled by insulin, hampers the anabolic effect of PTH on osseointegration in rat.

A study limitation is that we have not included a normoglycemic control to prove that PTH exerts its anabolic function in this study. However, for ethical reasons, and because there is plenty of evidence including from our previous research that PTH stimulates osseointegration of miniscrews in the medullary part of the tibia, ${ }^{27}$ we have avoided to include these groups. Under the premise of these limitations, our data suggest that insulin treatment cannot reverse the anabolic effect of PTH on osseointegration in type 1 diabetic rat models.

\section{Conclusion}

The results of this study demonstrate that the metabolic characteristics of the diabetic rats produced a condition unable to respond to PTH treatment, even when hyperglycemia was controlled with insulin. 


\section{Disclosure}

The authors claim to have no financial interest, either directly or indirectly, in the products or information listed in the article.

\section{ApProval}

The Medical University of Vienna Ethical Review Board for Animal Research approved the study protocol (GZ 66.009/0275-II/10b/2008).

\section{ACKnowledgments}

The authors are grateful to Roberto Plasenzotti for veterinarian support and the staff of the Institute of Biomedical Research for taking care of the animals.

\section{REFERENCES}

1. Zhang $P$, Imai $K$. The relationship between age and healthcare expenditure among persons with diabetes mellitus. Expert Opin Pharmacother. 2007;8:49-57.

2. Morley JE. Diabetes and aging: Epidemiologic overview. Clin Geriatr Med. 2008;24:395-405, v.

3. Herskind AM, Christensen K, Norgaard-Andersen K, et al. Diabetes mellitus and healing of closed fractures. Diabete Metab. 1992;18:63-64.

4. White CB, Turner NS, Lee GC, et al. Open ankle fractures in patients with diabetes mellitus. Clin Orthop Relat Res. 2003:37-44.

5. Hofbauer LC, Brueck CC, Singh SK, et al. Osteoporosis in patients with diabetes mellitus. J Bone Miner Res. 2007;22: 1317-1328.

6. Rakel A, Sheehy O, Rahme E, et al. Osteoporosis among patients with type 1 and type 2 diabetes. Diabetes Metab. 2008;34:193-205.

7. Retzepi M, Donos N. The effect of diabetes mellitus on osseous healing. Clin Oral Implants Res. 2010;21:673-681.

8. Javed F, Romanos GE. Impact of diabetes mellitus and glycemic control on the osseointegration of dental implants: A systematic literature review. J Periodontol. 2009;80:1719-1730.

9. Mombelli A, Cionca N. Systemic diseases affecting osseointegration therapy. Clin Oral Implants Res. 2006;17(suppl 2): 97-103.

10. Alsaadi G, Quirynen M, Michiles K, et al. Impact of local and systemic factors on the incidence of failures up to abutment connection with modified surface oral implants. J Clin Periodontol. 2008;35:51-57.

11. Quintero DG, Winger JN, Khashaba R, et al. Advanced glycation end products and rat dental implant osseointegration. J Oral Implantol. 2010;36: 97-103.

12. Oates TW, Dowell S, Robinson M, et al. Glycemic control and implant stabilization in type 2 diabetes mellitus. J Dent Res. 2009;88:367-371.

13. Neer RM, Arnaud CD, Zanchetta $\mathrm{JR}$, et al. Effect of parathyroid hormone (1-34) on fractures and bone mineral density in postmenopausal women with osteoporosis. N Engl J Med. 2001;344: 1434-1441.

14. Alkhiary YM, Gerstenfeld LC, Krall E, et al. Enhancement of experimental fracture-healing by systemic administration of recombinant human parathyroid hormone (PTH 1-34). J Bone Joint Surg Am. 2005; 87:731-741.

15. Andreassen TT, Ejersted C, Oxlund $\mathrm{H}$. Intermittent parathyroid hormone (1-34) treatment increases callus formation and mechanical strength of healing rat fractures. J Bone Miner Res. 1999; 14:960-968.

16. Kim HW, Jahng JS. Effect of intermittent administration of parathyroid hormone on fracture healing in ovariectomized rats. lowa Orthop J. 1999;19:71-77.

17. Komatsubara S, Mori S, Mashiba T, et al. Human parathyroid hormone (1-34) accelerates the fracture healing process of woven to lamellar bone replacement and new cortical shell formation in rat femora. Bone. 2005;36:678-687.

18. Manabe T, Mori S, Mashiba T, et al. Human parathyroid hormone (1-34) accelerates natural fracture healing process in the femoral osteotomy model of cynomolgus monkeys. Bone. 2007;40: 1475-1482.

19. Nakajima A, Shimoji N, Shiomi K, et al. Mechanisms for the enhancement of fracture healing in rats treated with intermittent low-dose human parathyroid hormone (1-34). J Bone Miner Res. 2002;17: 2038-2047.

20. Skripitz R, Aspenberg P. Implant fixation enhanced by intermittent treatment with parathyroid hormone. J Bone Joint Surg Br. 2001;83:437-440.

21. Aspenberg P, Genant HK, Johansson $\mathrm{T}$, et al. Teriparatide for acceleration of fracture repair in humans: A prospective, randomized, double-blind study of 102 postmenopausal women with distal radial fractures. J Bone Miner Res. 2010; 25:404-414.

22. Aspenberg P, Johansson T. Teriparatide improves early callus formation in distal radial fractures. Acta Orthop. 2010;81:234-236.

23. Bashutski JD, Eber RM, Kinney JS, et al. Teriparatide and osseous regeneration in the oral cavity. N Engl J Med. 2010; 363:2396-2405.

24. Shirota T, Tashiro M, Ohno K, et al. Effect of intermittent parathyroid hormone (1-34) treatment on the bone response after placement of titanium implants into the tibia of ovariectomized rats. J Oral Maxillofac Surg. 2003;61:471-480.

25. Kuchler U, Luvizuto ER, Tangl S, et al. Short-term teriparatide delivery and osseointegration: A clinical feasibility study. J Dent Res. 2011;90:1001-1006.

26. Kuchler U, Spilka T, Baron K, et al. Intermittent parathyroid hormone fails to stimulate osseointegration in diabetic rats. Clin Oral Implants Res. 2011;22:518-523.

27. Mair B, Tangl S, Feierfeil J, et al. Age-related efficacy of parathyroid hormone on osseointegration in the rat. Clin Oral Implants Res. 2009;20:400-405.

28. Fang $Y$, Wang LP, Du FL, et al. Effects of insulin-like growth factor I on alveolar bone remodeling in diabetic rats. J Periodontal Res. 2013;48:144-150.

29. Ishizuya $T$, Yokose $S$, Hori $M$, et al. Parathyroid hormone exerts disparate effects on osteoblast differentiation depending on exposure time in rat osteoblastic cells. J Clin Invest. 1997;99: 2961-2970.

30. Miyakoshi N, Kasukawa Y, Linkhart TA, et al. Evidence that anabolic effects of PTH on bone require IGF-I in growing mice. Endocrinology. 2001;142:43494356. 